\title{
Publisher Correction: Mutation-selection balance and compensatory mechanisms in tumour evolution
}

Erez Persi, Yuri I. Wolf(iD, David Horn, Eytan Ruppin (1D, Francesca Demichelis (1), Robert A. Gatenby, Robert J. Gillies and Eugene V. Koonin (1)

Correction to: Nature Reviews Genetics https://doi.org/10.1038/s41576-020-00299-4, published online 30 November 2020.

In the originally published online version of this article, the equation for the fixation probability $\left(P_{\text {fix }}\right)$ in Box 1 contained errors in the typesetting of $N_{e}$ (the effective population size). These errors have been corrected online. The editors apologize for these errors.

https://doi.org/10.1038/s41576-020-00313-9 I Published online 14 December 2020

(c) Springer Nature Limited 2020 